\title{
Performance of a Wireless Access Protocol on Correlated Rayleigh-Fading Channels with Capture
}

\author{
A. Chockalingam, Member, IEEE, Michele Zorzi, Senior Member, IEEE, \\ Laurence B. Milstein, Fellow, IEEE, and Pallapa Venkataram
}

\begin{abstract}
The throughput performance of a wireless media access protocol taking into account the effect of correlated channel fading, capture, and propagation delay is analyzed. For efficient access on the uplink (mobile-to-base-station link), the protocol makes use of the uplink channel status information which is conveyed to the mobiles through a busy/idle flag broadcast on the downlink (base-station-to-mobile link). A first-order Markov model is used to describe the correlation in the packet success/failure process on a Rayleigh-fading channel. The analytical results obtained through the first-order Markov approximation of the channel are compared to those obtained from an independent and identically distributed (i.i.d.) channel model. The Markovianfading channel model is shown to provide better performance results than the i.i.d. channel model. Simulations show that a first-order Markov approximation of the Rayleigh-fading process is quite accurate. An enhanced version of the access protocol to take advantage of the memory in the fading channel behavior is proposed and analyzed. The effect of retransmission of erroneous data packets and propagation delay on the throughput is also analyzed. It is shown that the access protocol with an error detect $(E D)$ feature is efficient in slow fading (e.g., pedestrian user speeds), whereas a retransmission protocol is more efficient in fast fading (e.g., vehicular user speeds).
\end{abstract}

Index Terms - Capture, correlated fading, Markov channel, retransmission, wireless access.

\section{INTRODUCTION}

$\mathbf{N}$ EXT-GENERATION wireless networks are envisaged to support high data rates, packet oriented transport, and multimedia traffic, and so the design and performance analysis of efficient media access protocols for integrated wireless

Paper approved by J. Chuang, the Editor for Wireless Networks of the IEEE Communications Society. Manuscript received March 5, 1997; revised August 25, 1997. This work was supported in part by TRW, by Airtouch, by the Center for Wireless Communications at the University of California-San Diego, and by the MICRO Program of the State of California. This paper was presented in part at the IEEE Global Communications Conference, London, U.K., November 1996.

A. Chockalingam is with Qualcomm, Inc., San Diego, CA 92121 USA (e-mail: achockal@qualcomm.com).

M. Zorzi is with the Center for Wireless Communications, University of California-San Diego, La Jolla, CA 92093-0407 USA (e-mail: zorzi@cwc.ucsd.edu).

L. B. Milstein is with the Department of Electrical and Computer Engineering, University of California-San Diego, La Jolla, CA 920930407 USA (e-mail: milstein@ece.ucsd.edu).

P. Venkataram is with the Department of Electrical Communication Engineering, Indian Institute of Science, Bangalore 560 012, India (e-mail: pallapa@ece.iisc.ernet.in).

Publisher Item Identifier S 0090-6778(98)03869-0. multimedia applications are gaining importance [1]-[3]. One important issue that most analyses in the literature ignore is the effect of channel fading on the protocol performance. In fact, mobile radio channels are severely affected by time-varying losses due to distance, shadowing (blockage due to buildings, trees, etc.), and multipath fading. While the variation in the losses due to distance and shadowing is relatively slow, the variation due to multipath fading is quite rapid [4], [5]. The fading envelope due to multipath often follows a Rayleigh distribution, so that the envelope squared (i.e., the power) has an exponential distribution [5]. Most notably, the correlation in the multipath fading behavior and its effect on the performance of access protocols have not been adequately addressed in the literature so far. The primary focus of this paper is to address this void.

In the past, most models for data block transmission (e.g., in data link protocols) have assumed that the block transmissions were independent and identically distributed (i.i.d.). Also, many protocols were designed for an i.i.d. channel, and techniques were developed to eliminate channel memory (e.g., by interleaving). A newer approach is to take advantage of the channel correlation (e.g., exploiting some prediction techniques) to obtain better performance, rather than destroying the memory. A natural way to model a channel with memory is to approximate it by means of a Markov model. Markov models to approximate Rayleigh-fading channels have been considered in [6] and [7]. In [8] the binary process which describes packet successes and failures on a Rayleigh-fading channel was investigated using mutual information, and it was shown that often a first-order Markov model is, in fact, an adequate approximation. In this paper we adopt a first-order Markov approximation to the fading channel model in order to investigate the effect of Rayleigh fading on the performance of an access protocol. The protocol we consider in this paper makes use of the uplink (mobile-to-base-station link) channel status information which is conveyed to the mobiles through the downlink (base-station-to-mobile link) broadcast from the base station by periodically inserting a busy/idle flag [9]. This protocol can be viewed as a hybrid protocol employing the slotted ALOHA and the busy tone sensing concepts [10]. A header packet is sent on a contention basis first, following which data packets are sent on a reservation basis. By this approach, packet losses due to collision are restricted to occur only among header packet transmissions. Also, as we 
will see, capture conditions [11] are better handled in this protocol by allowing the base station to send a nonbinary feedback to identify the successful mobile in the event of capture among simultaneous header packet transmissions from multiple mobiles.

The access protocol is described in Section II. The fading channel model, the packet success/failure process, and the parameters of the Markov approximation of the channel are presented in Section III. The throughput performance of the protocol is analyzed in Section IV, assuming instantaneous and error-free feedback. We also propose and analyze an enhanced version of the protocol-called the protocol with an error detect (ED) feature-which attempts to improve the performance by exploiting the memory in the fading channel behavior. The strategy here is that a user, upon packet error events being detected in its ongoing message transmission, is advised by the base station to abort the transmission and attempt later. This scheme is expected to give good results in the presence of significant channel burstiness, as it avoids insisting on transmission in slots which are likely to be in error, and lets other users (whose channel conditions might be good) access the channel. However, in the presence of rapidly varying channels, which result in low correlation between errors in consecutive slots, the strategy of the $E D$ protocol may be too wasteful, as it effectively reduces the message length and therefore decreases the overall efficiency. Another classic way of recovering errors in packet transmission is through retransmission. We analyze a parameterized retransmission strategy at the media access layer to recover erroneous data packets. In Section $\mathrm{V}$ we analyze the performance of these protocols when the feedback is not instantaneous; that is, when the delays due to propagation and processing are larger than the slot duration. Such scenarios are typical in high data rate wireless systems. Section VI provides the conclusions.

\section{WiRELESS ACCESS PROTOCOL}

The operation of the wireless access protocol considered in this section (we will refer to it as the basic protocol) is described as follows. The uplink (mobile-to-base-station link) channel is slotted to one packet duration. Transmission attempts are made by the mobiles only at the slot boundaries. Each message generated at the mobiles consists of two segments, namely, the header segment and the data segment. The header segment is one packet in length. It carries control information such as destination address, number of packets in the data segment, etc. The data segment represents the actual traffic. It consists of a random number of data packets. A busy/idle flag indicating the activity on the uplink is made available to the mobiles at the beginning of each slot. This flag is broadcast by the base station, once every slot, on the downlink (base-station-to-mobile link).

According to the basic protocol, once a mobile receives a message to be delivered to the base station, it first checks the status of the received busy/idle flag. If the flag is set to busy, the mobile refrains from making a transmission attempt. If the flag is set to idle, then the mobile makes a transmission attempt by first sending the header packet on the uplink slot.
If the header packet is received successfully (without packet loss due to collision or fading), the base station broadcasts the ID of the successful mobile (capturing mobile in the event of collision among header packets from different mobiles), and sets the flag to busy for the $k$ subsequent slots, where $k$ is the number of packets in the successful mobile's data segment. This allows only the successful mobile to send all of its data packets continuously in those $k$ slots. The base station resets the flag back to the idle status once the message transmission is completed. On the other hand, if the header packet is lost (due to collision or fading), the base station will not respond with a busy flag, but will continue sending the idle flag. This is an indication to the mobile that the header packet was lost, and so it has to reschedule the transmission attempt to a later time.

It can be seen that the packet transmissions, as per the above feedback mechanism (when error-free), can experience fading, interference, and noise during header transmission, whereas during data transmission only fading and noise (no interference) are experienced. Thus, in the case of error-free feedback to all mobiles, collisions and hence capture are possible only during the header packet transmission and not during the transmission of data packets. However, errors in the busy/idle flag reception would result in collisions, and hence packet losses, during the transmission of data packets as well.

\section{Correlated FAding Channel Model}

In order to analyze the performance of the access protocol in the presence of fading, we consider a frequency-nonselective (flat) multipath-fading channel whose analytical model is described in the following. In the literature the flat-fading channel is modeled as a multiplicative complex function $\alpha(t)$ which is adequately described as a random process. A popular model considers a complex Gaussian random process with a given mean and covariance function [5]. On the time scale of the fading variations, the process can be considered as stationary. Therefore, with no loss in generality, we will normalize its power to one. The real and imaginary axes can be chosen so that the mean $\mu=E[\alpha(t)]$ is real. Also, we consider the covariance function, defined as

$$
K(\tau)=E\left[(\alpha(t+\tau)-\mu)^{*}(\alpha(t)-\mu)\right]
$$

Note that if $\mu=0$, the envelope of $\alpha(t)$ is Rayleigh-distributed for any $t$, and the envelope squared has an exponential distribution. When $\mu>0$, the resulting fading envelope is Rician-distributed and accounts for the presence of a line-ofsight (LOS) component. When the LOS component is absent, or has negligible power, the Rician model degenerates into the Rayleigh one.

In a widely accepted model the Gaussian process is assumed to have a bandlimited nonrational spectrum given by [5]

$$
S(f)=S(0)\left[1-\left(\frac{f}{f_{D}}\right)^{2}\right]^{-1 / 2}, \quad \text { for }|f|<f_{D}
$$


and zero otherwise, where $f_{D}=V / \lambda$ is the Doppler bandwidth, $V$ is the mobile speed, and $\lambda$ is the carrier wavelength. This spectrum corresponds to the covariance function

$$
K(\tau)=J_{0}\left(2 \pi f_{D}|\tau|\right)
$$

whose physical meaning has been investigated in [4] and [5]. $J_{0}(\cdot)$ is the Bessel function of the first kind and of zeroth order. Note that the correlation properties of the fading process depend only on $f_{D}|\tau|$. When $f_{D}|\tau|$ is small (e.g., $<0.1$ ), the process is very correlated ("slow" fading); on the other hand, for larger values of $f_{D}|\tau|$ (e.g., $>0.2$ ), successive samples of the channel are almost independent ("fast" fading). For high data rates (i.e., small $\tau$ ), the fading process can typically be considered as slowly varying, at least for the usual values of the carrier frequency (900-1800 MHz) and for typical mobile speeds, so that the dependence between transmissions of consecutive packets of data cannot be neglected. In particular, the assumption that the successes/failures of data packets constitute an i.i.d. process is far from reality and may lead to incorrect results when used to evaluate the performance of wireless access protocols. Another approach, which accounts for dependence, is as follows.

The packet success/failure process is modeled as the outcome of a comparison of the instantaneous signal-to-noise ratio (SNR) to a threshold value $\mathrm{SNR}_{\mathrm{t}}$ : if it is above the threshold, the packet is successfully decoded with probability one; otherwise, the packet is lost with probability one. If $F$ is the value of the fading margin, the instantaneous SNR (taking into account the effect of fading) is given by $\mathrm{SNR}_{\mathrm{t}} \mathrm{F}\left|\alpha^{2}(\mathrm{t})\right|$. Hence, the binary process that describes packet successes/failures on the channel $\beta_{j}$ can be obtained by quantization of the squared magnitude of the complex Gaussian description with the threshold $1 / F$, i.e.,

$$
\beta_{j}= \begin{cases}0, & \text { if } v_{j}^{2}>1 / F \\ 1, & \text { if } v_{j}^{2} \leq 1 / F\end{cases}
$$

where $v_{j}=|\alpha(j T)|$ is the amplitude of the fading envelope at time $j T, T$ is the packet duration, and " 1 " stands for a packet failure. We describe the above success/failure process on a mobile radio channel by a first-order Markov model. The parameters of the Markov model can be determined based on the fading model and the characteristics of the communications scheme. The transition probability matrix that describes the channel is given by

$$
\mathbf{M}_{c}=\left(\begin{array}{cc}
p & 1-p \\
1-q & q
\end{array}\right)
$$

where $p$ and $1-q$ are the probabilities that the packet transmission in slot $j$ is successful, given that the transmission in slot $j-1$ was successful or unsuccessful, respectively. Given the matrix $\mathbf{M}_{c}$, the channel model is completely characterized. In particular, the steady-state probability $P_{E}$ that a packet error occurs due to fading and noise is

$$
P_{E}=\frac{1-p}{2-p-q} .
$$

Also, note that $(1-q)^{-1}$ represents the average length of a burst of errors, which is described by a geometric random variable. The parameters of the above Markov model can be found as [8]

$$
\begin{aligned}
P_{E} & =1-e^{-1 / F} \\
q & =1-\frac{Q(\theta, \rho \theta)-Q(\rho \theta, \theta)}{e^{1 / F}-1}
\end{aligned}
$$

where

$$
\theta=\sqrt{\frac{2 / F}{1-\rho^{2}}} .
$$

$\rho=J_{0}\left(2 \pi f_{D} T\right)$ is the correlation coefficient of two successive samples of the complex amplitude of a fading channel with Doppler frequency $f_{D}$ taken $T$ seconds apart, $f_{D} T$ is the normalized Doppler bandwidth, and

$$
Q(x, y)=\int_{y}^{\infty} e^{-\frac{\left(x^{2}+w^{2}\right)}{2}} I_{0}(x w) w d w
$$

is the Marcum $Q$ function. In (10) $I_{0}$ is the modified Bessel function of the first kind and of zeroth order. The Markov parameter $p$ can be obtained using (8) and (7) in (6). By choosing different values of $f_{D} T$, we can establish fading channel models with different degrees of correlation in the fading process.

\section{A. Header Packet Capture Probability}

Header packets from different mobiles can collide in a slot and the probability of capture under such conditions needs to be computed to carry out the throughput analysis. Consider simultaneous header packet transmissions from $n$ different mobiles in a slot, where the signal from user $j$ is received at the base station with power $\alpha_{j}^{2}, j=1,2, \cdots, n$. As stated earlier, in a multipath fading environment and in the absence of a LOS component, the $\alpha_{j}$ 's are Rayleigh-distributed and the $\alpha_{j}^{2}$ 's are exponentially distributed. Capture is said to occur in favor of user $i$ if

$$
\alpha_{i}^{2}>B\left[\sum_{j=1, j \neq i}^{n} \alpha_{j}^{2}+N_{0}\right]=B\left[\sum_{j=1, j \neq i}^{n} \alpha_{j}^{2}\right]+\frac{1}{F}
$$

where $B$ is defined as the capture threshold and $N_{0}$ is the noise power. ${ }^{1}$ From [12], the probability that there is a header packet success when $n$ simultaneously colliding header packet transmissions are present in a slot, $p_{s}^{(n)}$ can be found as

$$
p_{s}^{(n)}=n e^{-1 / F}\left(\frac{1}{1+B}\right)^{n-1} .
$$

From (12), there is no capture when $B \rightarrow \infty$ (i.e., $p_{s}^{(n)}=0$ for $n>1$ ). Also, $B=1$ corresponds to an idealized perfect capture condition [11].

\footnotetext{
${ }^{1}$ Note that, since in our model all powers are normalized so that $E\left[\alpha_{j}^{2}\right]=$ $1 \forall j, 1 / N_{0}$ is the average SNR and $1 /\left(B N_{0}\right)$ is the uplink fading margin.
} 


\section{Throughrut AnALYsis}

To analyze the system throughput, we initially assume that the feedback from the base station (busy/idle flag and the successful mobile ID) on the downlink is received instantaneously and error-free by all mobiles. In the subsequent analysis in Section V we will relax the instantaneous feedback assumption. The error-free feedback assumption is reasonable because the feedback consists of only a few bits, which can be provided with adequate error protection. We also make the following assumptions on the message arrival process and the message length distribution: 1) the message arrival process at each mobile is Bernoulli with rate $\lambda$ per slot (i.e., there are no arrivals or one arrival with probabilities $1-\lambda$ and $\lambda$, respectively) and 2) the length of the data segment of the message (not including the header packet) $k$, measured in integer number of packets, follows a geometric distribution with parameter $g_{d}\left(0<g_{d}<1\right)$ and probability mass function

$$
P[k=y]=\left\{\begin{array}{l}
g_{d}\left(1-g_{d}\right)^{y-1}, \quad y=1,2,3, \cdots \\
0, \quad \text { otherwise. }
\end{array}\right.
$$

For the Bernoulli arrival process, the probability that $n$ out of $N$ mobile users $(0 \leq n \leq N)$ make a transmission attempt in a slot $p_{n}$ is given by

$$
p_{n}=\left(\begin{array}{c}
N \\
n
\end{array}\right) \lambda^{n}(1-\lambda)^{N-n} .
$$

We first analyze the performance of the basic protocol described in Section II. Later, we extend the analysis to both an enhanced version of the protocol with an $E D$ feature that attempts to exploit the channel memory for better performance, as well as a retransmission protocol to recover erroneous data packets.

\section{A. Basic Protocol Performance}

Based on the assumptions described above, the evolution of the basic protocol on a Markovian channel can be tracked by means of a Markov chain with a finite number of states. An adequate state space consists of just five states describing the system status in a slot, namely: 1$)$ idle $(I) ; 2)$ header packet success $\left.\left(H_{s}\right) ; 3\right)$ header packet failure $\left(H_{f}\right)$; 4$)$ data packet success $\left(D_{s}\right)$; and 5) data packet failure $\left(D_{f}\right)$. Once the state transition probabilities of the chain $P_{i j}, i, j \in \Omega$, where $\Omega$ is the state space, are determined, the steady-state probability vector $\pi$ is given by the solution of the equations

$$
\pi=\pi \mathbf{P}, \quad \sum_{j \in \Omega} \pi_{j}=1 .
$$

The state transition probability matrix for the basic protocol $\mathbf{P}_{\text {basic }}$ can be written as

$$
\mathbf{P}_{\text {basic }}=\left[\begin{array}{ccccc}
X_{0} & X_{1} & X_{2} & 0 & 0 \\
0 & 0 & 0 & p^{\prime} & 1-p^{\prime} \\
X_{0} & X_{1} & X_{2} & 0 & 0 \\
g_{d} X_{0} & g_{d} X_{1} & g_{d} X_{2} & X_{3} & X_{4} \\
g_{d} X_{0} & g_{d} X_{1} & g_{d} X_{2} & X_{5} & X_{6}
\end{array}\right]
$$

where $X_{0}=p_{0}=(1-\lambda)^{N}, X_{1}=\sum_{i=1}^{N} p_{i} p_{s}^{(i)}=(1-$ $\left.P_{E}\right) p_{1}(1+x)^{N-1}, x=(\lambda) /((1+B)(1-\lambda)), X_{2}=1-$
$X_{0}-X_{1}, X_{3}=\left(1-g_{d}\right) p, X_{4}=\left(1-g_{d}\right)(1-p), X_{\tilde{5}}=$ $\left(1-g_{d}\right)(1-q)$, and $X_{6}=\left(1-g_{d}\right) q$. The arrival probabilities $p_{i}$ and the capture probabilities $p_{s}^{(i)}$ are given in (14) and (12), respectively. Note that in order to precisely track the transitions from header success state $\left(H_{s}\right)$ to data success state $\left(D_{s}\right)$ one would need to increase the number of states to take into account all of the possible numbers of simultaneously transmitting users in the header success state, on which the probability of having a data packet success in the next slot depends. Here, in order to have a smaller number of states, we assume that a transition from header success state to data success state occurs with probability $p^{\prime}$. In particular, we will consider $p^{\prime}=1$ as an optimistic approximation, and $p^{\prime}=p$ as a pessimistic approximation. We show that the throughput estimates obtained from these two approximations are tight, and they closely agree with the actual throughput values obtained by simulation. In all numerical results we assume $p^{\prime}=p$ so that the results presented are to be considered as conservative estimates.

Working through the steady-state analysis of the system, we obtain the expression for the average throughput of the basic protocol, in closed-form, as

$$
\eta_{\text {basic }}=\frac{X_{1}}{g_{d}+X_{1}}\left[\frac{g_{d} p^{\prime}+\left(1-g_{d}\right)(1-q)}{1+\left(1-g_{d}\right)(1-p-q)}\right] .
$$

Note that, since $X_{1} /\left(g_{d}+X_{1}\right)$ is a monotonically increasing function of $X_{1}$, and since $X_{1}$ is the only quantity in (17) which depends on $\lambda$, the value of $\lambda$ which maximizes $X_{1}$ also maximizes the throughput. In fact, the maximum throughput $\eta_{\max }$ occurs at the arrival rate $\lambda_{\max }$, given by

$$
\lambda_{\max }=\frac{1+B}{B N}
$$

which is independent of $p, q$ and $g_{d}$. Note that when there is no capture (i.e., $B \rightarrow \infty$ ), $\lambda_{\max }$ becomes equal to $1 / N$. When there is perfect capture (i.e., $B=1$ ), $\lambda_{\max }$ becomes equal to $2 / N$. In (17) successful header packet transmissions are not considered to be useful in the throughput computation so that $\eta_{\text {basic }}=\pi_{4}$, where $\pi_{4}$ is the steady-state probability of state $D_{s}$. The protocol throughput for an i.i.d. channel model can be derived by setting $p=p^{\prime}=1-q=1-P_{E}$ in (17), to obtain

$$
\eta_{\text {iid }}=\frac{X_{1}}{g_{d}+X_{1}}\left(1-P_{E}\right) \text {. }
$$

\section{B. Enhanced Protocol with ED Feature}

In the basic protocol described and analyzed above we allowed the mobile to continuously transmit all of the packets in the data segment of the message even when one or more of those packets were lost due to channel fading. However, the memory in the fading process can be exploited to modify the data transmission strategy by using the knowledge about the channel status information. As an example, consider the following. Under slow-fading conditions (where events in successive slots are expected to be highly correlated), the fact that the data packet in the current slot is received in error implies that the packet in the subsequent slot will also be received in error with high probability. Therefore, the protocol 
rules can be modified so that, when the base station detects such a "bad" channel condition during transmission of a data segment from a mobile, it could ask that mobile to abort transmission and release the channel. This avoids the likely occurrence of subsequent errors and allows other mobiles (which may, on the other hand, experience "good" channel conditions) to transmit.

To investigate how this idea can be used to enhance the protocol performance, we analyze a simple modified version of the basic protocol. We refer to the modified scheme as the protocol with an $E D$ feature. According to the $E D$ protocol, if a packet in the data segment of the message is received in error, the base station sends out an idle flag in the next slot (instead of sending a busy flag in all $k$ data slots, as the basic protocol would do) to prompt the mobile to terminate the ongoing data transmission. Such a strategy enables other mobiles to access the channel during those slots which otherwise could have witnessed, with high probability, loss of packets due to fading.

Note that the transition probability matrix for the ED protocol $\mathbf{P}_{\mathrm{ED}}$ will be the same as that of the basic protocol [matrix $\mathbf{P}_{\text {basic }}$, given by (16)], except for the transition probabilities from state $D_{f}$. In fact, for the $E D$ protocol, the transition probabilities from state $D_{f}$ will be the same as those from the idle state. Accordingly, the transition probability matrix for the $E D$ protocol is given by

$$
\mathbf{P}_{\mathrm{ED}}=\left[\begin{array}{ccccc}
X_{0} & X_{1} & X_{2} & 0 & 0 \\
0 & 0 & 0 & p^{\prime} & 1-p^{\prime} \\
X_{0} & X_{1} & X_{2} & 0 & 0 \\
g_{d} X_{0} & g_{d} X_{1} & g_{d} X_{2} & X_{3} & X_{4} \\
X_{0} & X_{1} & X_{2} & 0 & 0
\end{array}\right]
$$

where $X_{0}, X_{1}, X_{2}, X_{3}$, and $X_{4}$ are as defined in Section IVA. From (20), the throughput expression for the $E D$ protocol can be derived as

$$
\eta_{\mathrm{ED}}=\frac{p^{\prime} X_{1}}{1-\left(1-g_{d}\right) p+X_{1}\left[1-\left(1-g_{d}\right)\left(p-p^{\prime}\right)\right]}
$$

which, again, is maximum for $\lambda=(1+B) /(B N)$.

\section{Protocol with Retransmission of Erroneous Data Packets}

The basic protocol does not take any action in the event of data packet errors, i.e., data packets which are corrupted during transmission are just lost and the recovery of such errors are left to higher layer protocols. The ED protocol described in the previous subsection, on the other hand, reacts to packet errors by aborting the ongoing message transmission. However, in the presence of rapidly varying channels, which result in low correlation between errors in consecutive slots, the strategy of the $E D$ protocol may be too wasteful, as it effectively reduces the message length and therefore decreases the overall efficiency. Another classic way of recovering errors in packet transmission is through retransmission. Instead of ignoring packet errors (as in the basic protocol) or aborting the message transmission altogether (as in the $E D$ variation), a packet is retransmitted if it is received in error. In the local wireless environment under consideration, where the feedback is assumed to be instantaneous, a packet in error can be retransmitted in the immediately following slot. In this case the base station would need to send a nonbinary feedback (busy/idle/retransmit) in order to avoid a collision among a retransmission packet and header packets from other users. Thus, with the retransmission strategy, a geometric length message of $L$ packets (with $E[L]=1 / g_{d}$ ) will take $L^{\prime}$ slots to finally get through, due to possible retransmissions. Therefore, we will have

$$
L^{\prime}=\sum_{j=1}^{L} Y_{j}
$$

where $Y_{j}$ is an integer random variable equal to the number of transmissions it takes packet $j$ to be successfully received. Note that the first transmission of data packet $j$ has a probability of success $p$ for $j>1$. This is because the first transmission of packet $j$ immediately follows the successful transmission of packet $j-1$. On the other hand, if the transmission is unsuccessful, then the probability that the immediately following retransmission succeeds is $1-q$. Thus, for $j>1$, the random variable $Y_{j}$ has the following probability mass function:

$$
P\left[Y_{j}=y\right]=\left\{\begin{array}{l}
p, \quad y=1 \\
(1-p) q^{y-2}(1-q), \quad y>1 .
\end{array}\right.
$$

The same argument applies for $j=1$ as well, but the probability $p^{\prime}$ is to be used instead of $p$ in this case.

Since the use of retransmissions occurs only when a data packet is in error, the transition matrix for the protocol is the same as for the basic protocol except for the last row (transitions from state $D_{f}$, which corresponds to a data packet in error). In fact, after an erroneous data packet, a retransmission attempt is always performed and therefore only transitions to $D_{s}$ (with probability $1-q$ ) or $D_{f}$ (with probability $q$ ) are allowed (in other words, a message cannot end with a failed transmission). Therefore, the transition probability matrix for the retransmission protocol can be written as

$$
\mathbf{P}_{\text {retx }}=\left[\begin{array}{ccccc}
X_{0} & X_{1} & X_{2} & 0 & 0 \\
0 & 0 & 0 & p^{\prime} & 1-p^{\prime} \\
X_{0} & X_{1} & X_{2} & 0 & 0 \\
g_{d} X_{0} & g_{d} X_{1} & g_{d} X_{2} & X_{3} & X_{4} \\
0 & 0 & 0 & 1-q & q
\end{array}\right] .
$$

From the above, the throughput expression for the retransmission protocol can be derived as

$$
\eta_{\text {retx }}=\frac{(1-q) X_{1}}{g_{d}(1-q)+X_{1}\left[2-p-q+g_{d}\left(p-p^{\prime}\right)\right]}
$$

which also results in maximum throughput when $\lambda=$ $(1+B) /(B N)$. In the case of $p^{\prime}=p$, (25) can be further simplified as

$$
\eta_{\mathrm{retx}}=\frac{p_{1}\left(1-P_{E}\right)(1+x)^{N-1}}{g_{d}+p_{1}(1+x)^{N-1}}
$$

where $x=(\lambda) /((1+B)(1-\lambda))$, and $p_{1}$ is obtained from (14) for $n=1$. From (26), it can be seen that the retransmission protocol throughput remains independent of the Markov parameters of the channel $p$ and $q$. 
Further, instead of terminating the data transmission at the first instance of a data packet failure (as in the $E D$ protocol) or repeatedly sending a data packet until success (as in the retransmission protocol), the base station could allow the mobile to resend a lost data packet only a limited number of times (defined as a parameter $n_{r}$ ), after which the mobile is asked to abort the data transmission. As can be seen, this is a generalized form of the protocol, and both $E D$ and retransmission protocols can be thought of as special cases of this generalized form for $n_{r}=0$ and $n_{r} \rightarrow \infty$, respectively. The transition probabilities for the generalized protocol can be written exactly as for the $E D$ protocol, except that the single $D_{f}$ state in the $E D$ case is expanded into $\left(n_{r}+1\right)$ different states; that is, $D_{f}(0), D_{f}(1), \cdots, D_{f}\left(n_{r}\right)$, where $D_{f}(j)$ corresponds to failure of the $(j+1)$ th transmission of a data packet. Accordingly, the state transition probability matrix for the parametrized retransmission strategy can be written as follows:

$\mathrm{P}_{\text {retx }}^{\prime}$
$=\left[\begin{array}{cccccccccc}X_{0} & X_{1} & X_{2} & 0 & 0 & 0 & 0 & . & \cdot & 0 \\ 0 & 0 & 0 & p^{\prime} & 1-p^{\prime} & 0 & 0 & . & . & 0 \\ X_{0} & X_{1} & X_{2} & 0 & 0 & 0 & 0 & . & . & 0 \\ g_{d} X_{0} & g_{d} X_{1} & g_{d} X_{2} & X_{3} & X_{4} & 0 & 0 & . & . & 0 \\ 0 & 0 & 0 & 1-q & 0 & q & 0 & . & . & 0 \\ 0 & 0 & 0 & 1-q & 0 & 0 & q & . & . & 0 \\ . & . & . & . & . & . & . & . & . & . \\ . & . & . & . & . & . & . & . & . & . \\ 0 & 0 & 0 & 1-q & 0 & 0 & 0 & . & \cdot & q \\ X_{0} & X_{1} & X_{2} & 0 & 0 & 0 & 0 & . & . & 0\end{array}\right]$.

\section{Results and Discussion}

In Fig. 1, the throughput performance of the different versions of the access protocol obtained from (17), (19), (21), and (25) is plotted versus the message arrival rate $\lambda$ under no capture condition (i.e., $B \rightarrow \infty$ ) and for $N=10$ users. A $g_{d}$ value of 0.1 , corresponding to an average message length of ten packets per message (not including the header), is used. Plots are shown for uplink fading margins $F$ of 5 and $10 \mathrm{~dB}$. For the basic, ED, and retransmission protocols, a normalized Doppler bandwidth $f_{D} T$ of 0.02 (representing slow fading) is chosen. The extreme case of i.i.d. packet errors with the same marginal error rate is also plotted for comparison. The effect of varying the normalized values of the Doppler bandwidth $f_{D} T$, the uplink fading margin $F$, and the average message length $1 / g_{d}$ is illustrated in the subsequent graphs (Figs. 2-4) for the case of no capture. In Figs. 1-4 the solid lines represent the analytical results obtained from the throughput expressions, whereas the markers represent simulation points. As mentioned earlier, in computing the analytical results we computed the throughput by choosing $p^{\prime}=p$. This is a good approximation for all of the protocols considered because, over a range of values of different parameters, the estimated throughputs were found to be at most $2 \%-2.5 \%$ worse than optimistic throughputs obtained by using $p^{\prime}=1$. Further, the results obtained by explicit simulation of the protocols on a correlated fading channel are seen to be in close agreement with the

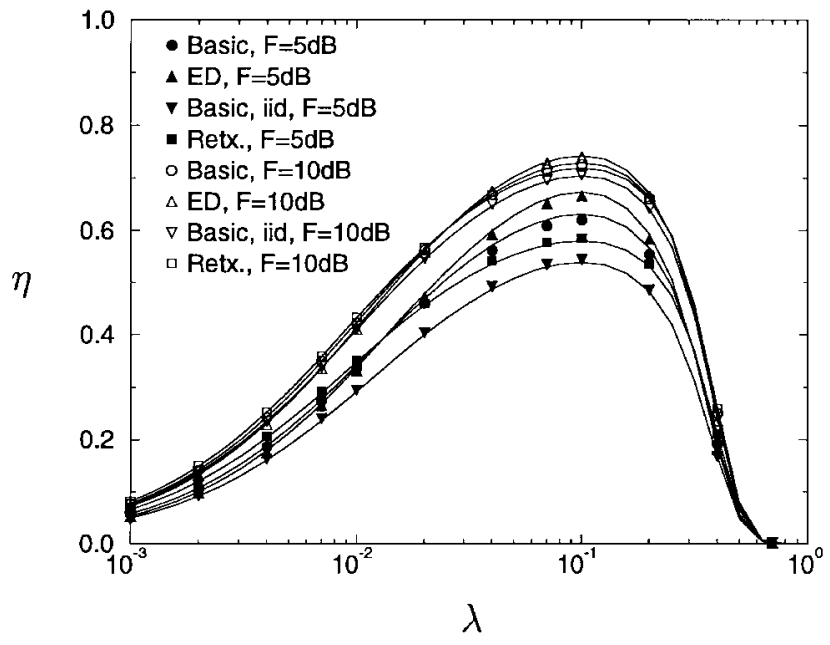

Fig. 1. Throughput $\eta$ versus message arrival rate $\lambda . N=10, g_{d}=0.1$, $f_{D} T=0.02$, no capture, instantaneous and error-free feedback.

analytical results. The correlated Rayleigh-fading channel was simulated using the method proposed by Jakes [5].

From Fig. 1 we observe the following. As was analytically computed, for all three protocol schemes ( $b a$ sic/ED/retransmission) and both channel models (slow fading/i.i.d.), the maximum achievable throughput occurs when $\lambda=1 / N$, and this gives good robustness against either possible variations or wrong estimates of the channel parameters. Even with a small uplink fading margin of $5 \mathrm{~dB}$, the basic protocol is found to offer a maximum throughput of about 0.631 successful data packets per slot. The ED protocol is found to perform better than both the basic and the retransmission protocols (e.g., maximum throughput of 0.673 for the $E D$ protocol against 0.631 for the basic protocol and 0.579 for the retransmission protocol). This was to be expected, because the fading rate considered is small (i.e., $f_{D} T=0.02$ ), and the basic protocol allows the mobiles to transmit all of the data packets in a message without any break, even under deep-fade situations. On the other hand, if a mobile encounters a deep fade during its data segment transmission, the $E D$ protocol releases the channel from that mobile and allows other mobiles to access the channel, thereby increasing the channel utilization efficiency. Further, for the considered $f_{D} T$ value of 0.02 , it is also reasonable for the retransmission protocol to perform more poorly than both the basic and the $E D$ protocols because the high burstiness of the packet errors at $f_{D} T=0.02$ would cause the retransmission protocol to use more slots for resending the erroneous packets. However, at high values of $f_{D} T$, as seen from Fig. 2, the retransmission protocol performs better than the basic and $E D$ protocols. When the uplink fading margin is $10 \mathrm{~dB}$, the relative performance of all three protocols remains the same but the actual differences in performance become smaller. For example, the maximum throughput for the basic, ED, and retransmission protocols are 0.728, 0.742, and 0.704, respectively.

A comparison between the performance predictions of an i.i.d. channel model and the Markov-fading model is also made in Fig. 1. It is seen that the i.i.d. model provides 


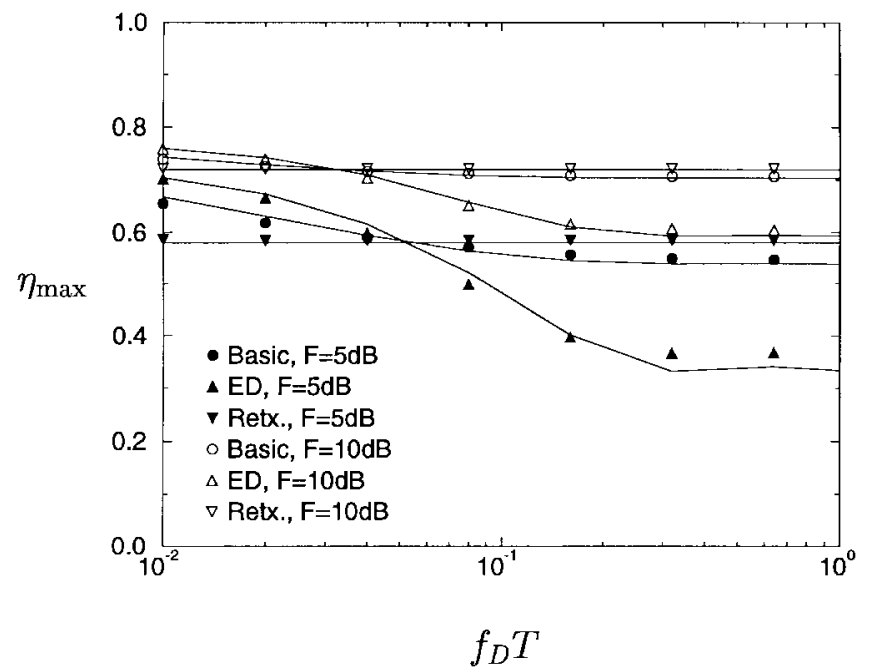

Fig. 2. Maximum throughput $\eta_{\max }$ versus normalized Doppler bandwidth $f_{D} T . N=10, g_{d}=0.1$, no capture, $\lambda=1 / N$, instantaneous and error-free feedback.

a pessimistic estimate of the throughput performance compared to the Markov model under slow-fading conditions (i.e., $\left.f_{D} T=0.02\right)$. However, it has been found that for fast-fading conditions (e.g., $f_{D} T=1$ ), both the i.i.d. and Markov models tend to produce close performance results. It is further noted that the first-order Markov approximation of the fading process is quite accurate, since the results computed through Markov analysis closely agree with those obtained by direct simulation of the protocol and the fading process.

Fig. 2 illustrates the effect of varying $f_{D} T$ on the maximum achievable throughput for the basic, ED, and retransmission protocols at $g_{d}=0.1, N=10, B \rightarrow \infty$, and $\lambda=1 / N$. As seen earlier, the $E D$ protocol performs better than both the basic and the retransmission protocols for small values of $f_{D} T$ (e.g., $<0.08$ for $F=5 \mathrm{~dB}$ ), whereas at high values of $f_{D} T$ (i.e., for weaker correlation between packet errors), the retransmission protocol performs better than the basic and $E D$ protocols. This performance crossover is due to the fact that at high values of $f_{D} T$, each packet during the data segment transmission would experience a nearly i.i.d. success/failure event, and terminating the data transmission in the event of a single packet failure (as done in the $E D$ protocol) reduces the average number of packet success events during the data segment transmission. This observation suggests that the $E D$ protocol is suited for networks supporting many slowly moving users (e.g., local area environment) and that the retransmission protocol is more suited for networks supporting many fast-moving users (e.g., cellular environment).

Fig. 3 shows the effect of varying the uplink fading margin $F$ on the throughput performance of the protocols when $N=10, g_{d}=0.1, B \rightarrow \infty, \lambda=1 / N$, and $f_{D} T=0.02$. At low fading margins, the $E D$ protocol performs markedly better than the other protocols. At high fading margins (e.g., $>15 \mathrm{~dB}$ ), all of the protocols tend to perform almost the same. However, over a typical range of uplink fading margins (5-10 dB), the ED protocol shows noticeable improvements over the basic and retransmission protocols under slow-fading conditions. Fig. 4 shows the effect of increasing the average

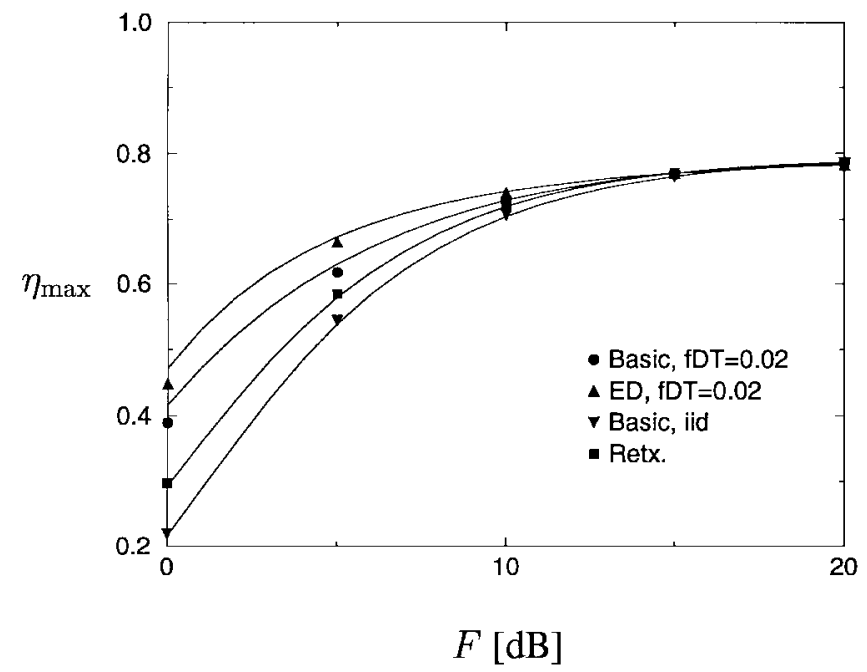

Fig. 3. Maximum throughput $\eta_{\max }$ versus uplink fading margin $F$ in $\mathrm{dB}$. $N=10, g_{d}=0.1$, no capture, $\lambda=1 / N, f_{D} T=0.02$, instantaneous and error-free feedback.

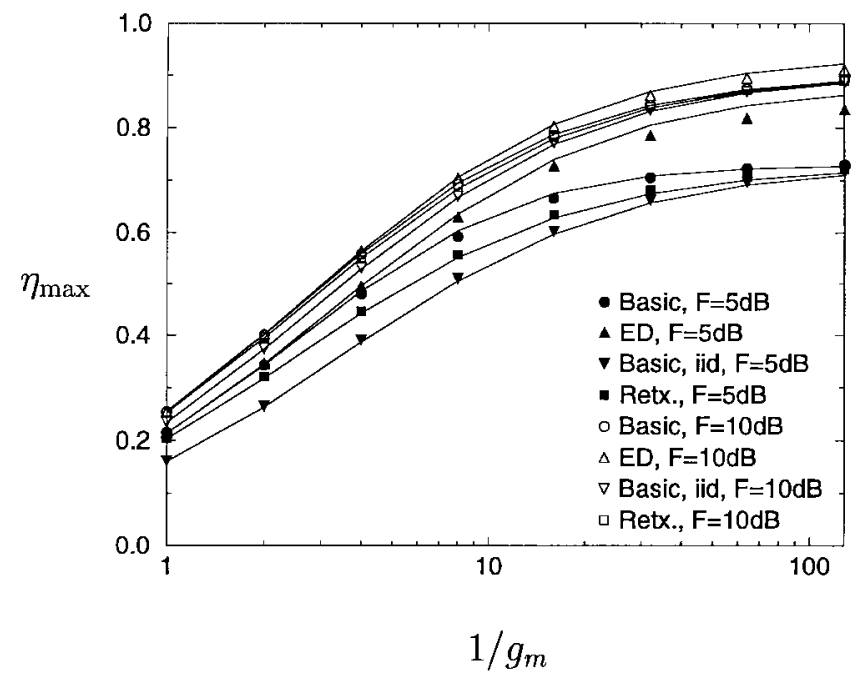

Fig. 4. Maximum throughput $\eta_{\max }$ versus average message length $1 / g_{d}$. $N=10, f_{D} T=0.02$, no capture, $\lambda=1 / N$, instantaneous and error-free feedback.

message length $1 / g_{d}$ on the throughput. It can be seen that the throughput improves for smaller values of $g_{d}$, i.e., for longer message lengths. This suggests that the protocol is suitable for messaging applications like file transfers, etc. However, the increased throughput for large message sizes will come at the expense of increased delay performance of the protocols [13].

The effect of header packet capture on the maximum throughput performance of all of the protocols is illustrated in Fig. 5 for fading margins of 5 and $10 \mathrm{~dB}$ at an $f_{D} T$ value of 0.02 . In the case of the basic protocol with $5-\mathrm{dB}$ fading margin, the maximum achievable throughput increases from 0.631 under no capture (e.g., $B=30 \mathrm{~dB}$ ) to 0.726 under perfect capture (i.e., $B=0 \mathrm{~dB}$ ). This is about a $15 \%$ increase in the maximum achievable throughput due to the header packet capture phenomenon. For the same set of conditions, the $E D$ protocol resulted in an $18 \%$ increase ( 0.673 to 0.795$)$ in 


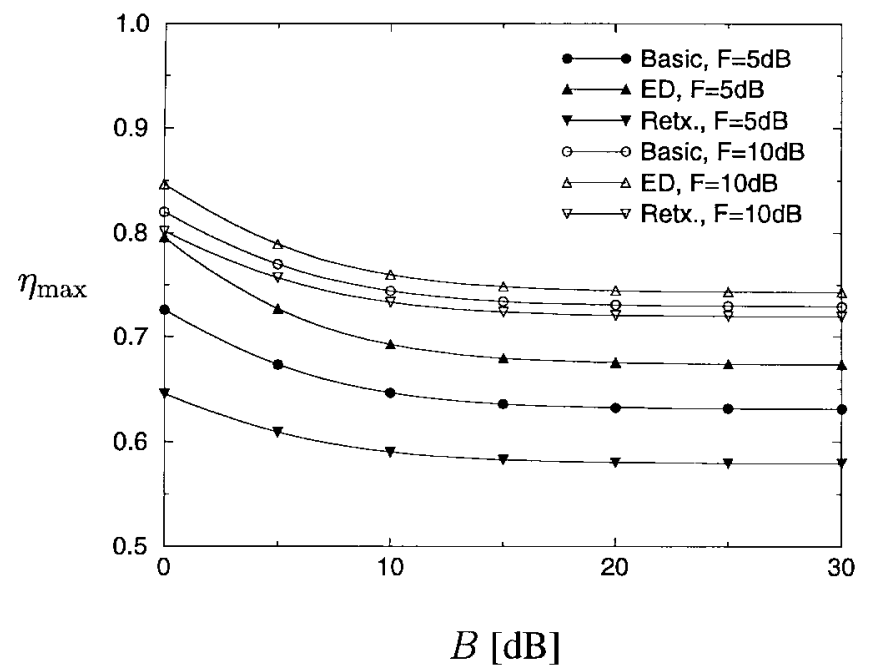

Fig. 5. Maximum throughput $\eta_{\max }$ versus capture threshold $B$ in $\mathrm{dB}$ $N=10, g_{d}=0.1, \lambda=(1+B) / B N, f_{D} T=0.02$, instantaneous and error-free feedback.

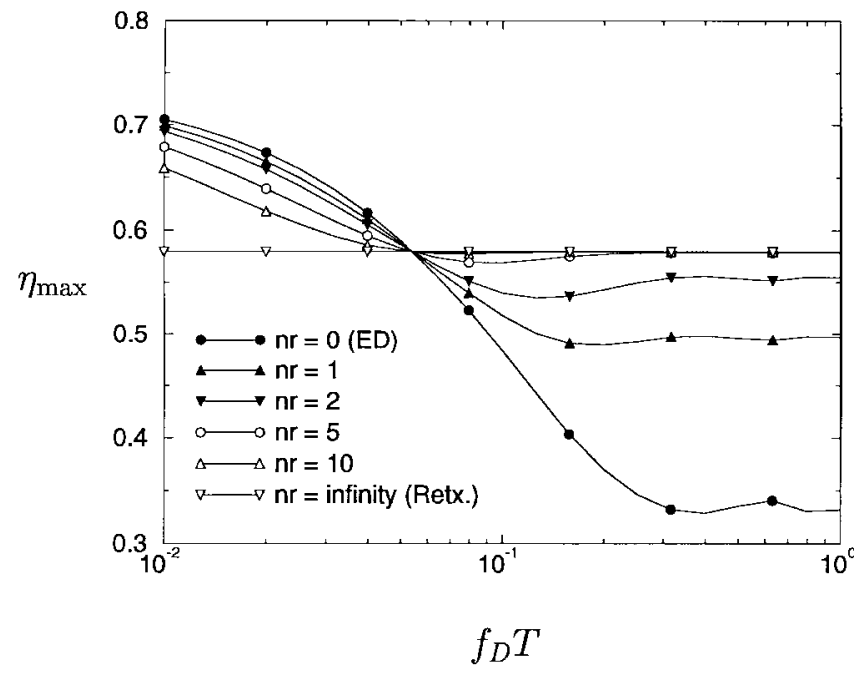

Fig. 6. Maximum throughput $\eta_{\max }$ versus normalized Doppler bandwidth $f_{D} T$. Parameterized retransmission $\left(n_{r}=0,1,2,5,10, \infty\right), N=10$, $g_{d}=0.1, F=5 \mathrm{~dB}, \lambda=1 / N$, no capture, instantaneous and error-free feedback.

maximum throughput and the retransmission protocol resulted in an increase of about $11 \%$ (0.579 to 0.645$)$.

In Fig. 6, maximum throughput performance curves are plotted for the parameterized retransmission strategy as a function of $f_{D} T$ for $F=5 \mathrm{~dB}$ and no capture. These curves are obtained by solving (27) for different values of the parameter $n_{r}$ (i.e., $1,2,5,10$ ). The performance of $E D$ and retransmission protocols are also plotted as limiting cases when $n_{r}=0$ and $n_{r} \rightarrow \infty$, respectively. As observed earlier, the $E D$ protocol performs best when the fading is slow and worst when fading is fast. Under fastfading conditions (e.g., $f_{D} T>0.1$ ), it is interesting to see that the performance improves significantly compared to the $E D$ protocol even if only one retransmission $\left(n_{r}=1\right)$ is allowed for the lost data packet recovery. In fact, just two or three retransmissions are adequate to establish almost the same performance as the retransmission protocol under fast- fading conditions. Even in slow fading $\left(f_{D} T<0.1\right)$, the parameterized retransmission strategy performs well, close to the ED protocol's performance, which is best in slow fading. In summary, a parameterized retransmission strategy which allows a maximum of two or three retransmissions is found to result in good performance over the range of mobile speeds of interest.

We further note here that in all of the analyses presented above, it has been assumed that when a header packet error occurs, the header is simply dropped and the message is regenerated as a new arrival according to a Bernoulli process. A more comprehensive analytical model which considers retransmission of lost header packets by allowing the mobile users to be either in a backlogged state or nonbacklogged state is studied in [13]. The results in [13] are qualitatively similar to those presented in this paper.

\section{Effect of Propagation and Processing Delays}

The instantaneous feedback (i.e., zero propagation and processing delay) assumed in the previous section can be valid in situations where the delays due to propagation and processing are very small compared to the slot duration. However, this assumption may not be always valid, particularly when high data rate transmissions are considered. In this section we analyze the performance of the basic, $E D$, and retransmission protocols when the feedback is not instantaneous. Analyzing the protocol performance for any general value of delay, when it spans multiple slot intervals, appears difficult (the state space quickly becomes too large). However, in local wireless environments propagation delays are small so that consideration of propagation and processing delays spanning at most one slot interval seems adequate. Here, we derive approximate expressions for the throughput performance when the propagation and processing delay spans one slot interval. Simulations show that the approximate analytical predictions are fairly accurate.

In the case of nonzero propagation and processing delay, following a header packet transmission, the mobile waits for the busy/idle feedback from the base station. It does not send any data packet in the slots that elapse between the header transmission and the receipt of the feedback. These vulnerable slots are sensed as idle by other mobiles which may send their header packets in those slots. However, if the feedback received immediately following the vulnerable slot(s) is a busy flag along with the successful mobile ID, then the mobiles which sent header packets in the vulnerable slot(s) will stop and attempt later. The successful mobile will then send the data packets following the rules of the particular protocol. In the case of the basic protocol, the base station would set the $k$ slots following the vulnerable slot(s) as busy, over which the mobile sends all of its $k$ data packets. In the case of the $E D$ protocol, if the base station encounters a packet error in the ongoing data transmission, it sends out an idle flag, thus requiring the mobile to terminate the data transmission. However, because of the delay, there will be a time lag (equal to the number of vulnerable slots) in the actual termination of data transmission at the mobile. In the case of the retransmission protocol, for 
each data packet that was received in error, the base station sends out a retransmit flag to enable recovery of those lost data packets. In addition, the base station has to force the slot next to the last data packet (i.e., the $k$ th data packet, where $k$ is the number of packets in the data segment) transmission slot to go unused (i.e., nobody is allowed to send header packets in that slot). This has to be done because of the time lag experienced by the mobile in knowing whether it has to retransmit the last data packet or not. Thus, in the retransmission protocol the slot following the successful last data packet slot is always wasted.

In the following we provide the throughput analysis for the case where the number of vulnerable slots is one. To make the analysis tractable, we make the following simplifying assumption. Even if there is no header success in the slot prior to a vulnerable slot, the other mobiles which sent header packets in the vulnerable slot are instructed by the base station to stop immediately and postpone their attempts to a later time, thus ignoring a possible header success in the vulnerable slot. By forcing this constraint, the slot following the vulnerable slot is either used for data packet transmission by a mobile whose header succeeded in the slot prior to the vulnerable slot, or is available as an idle slot for all of the mobiles to contend.

With the above assumption, the following seven different states are adequate to represent the state space for the basic protocol: 1) idle; 2) header packet success; 3) header packet failure; 4) vulnerable slot following a header success; 5) vulnerable slot following a header failure; 6) data packet success; and 7) data packet failure. From the header packet success state (state 2), the system moves to state 4 with probability one. Likewise, the system moves from state 3 to state 5 with probability one. The probability of a data packet success/failure in the slot next to the vulnerable slot is obtained as the two-step transition probability from the slot in which the header was sent, given by the appropriate entry of $\mathbf{M}_{c}^{2}$. In other words, from state 4 , the system moves to states 6 and 7 with probabilities $X_{7}=p^{2}+(1-p)(1-q)$ and $X_{8}=p(1-p)+(1-p) q$, respectively. Note that state 4 does not specify the channel status in the vulnerable slot so that the most recent information available about the channel relates to the previous slot. Thus, the state transition probability matrix for the basic protocol with one-slot propagation and processing delay $P_{\text {basic }}^{(d)}$ can be written as

$$
\mathbf{P}_{\text {basic }}^{(d)}=\left[\begin{array}{ccccccc}
X_{0} & X_{1} & X_{2} & 0 & 0 & 0 & 0 \\
0 & 0 & 0 & 1 & 0 & 0 & 0 \\
0 & 0 & 0 & 0 & 1 & 0 & 0 \\
0 & 0 & 0 & 0 & 0 & X_{7} & X_{8} \\
X_{0} & X_{1} & X_{2} & 0 & 0 & 0 & 0 \\
g_{d} X_{0} & g_{d} X_{1} & g_{d} X_{2} & 0 & 0 & X_{3} & X_{4} \\
g_{d} X_{0} & g_{d} X_{1} & g_{d} X_{2} & 0 & 0 & X_{5} & X_{6}
\end{array}\right]
$$

and the throughput is obtained as

$$
\eta_{\mathrm{basic}}^{(d)}=\pi_{6}
$$

In the case of the $E D$ protocol, states $1-5$ remain the same as states 1-5 in the basic protocol state space. However,

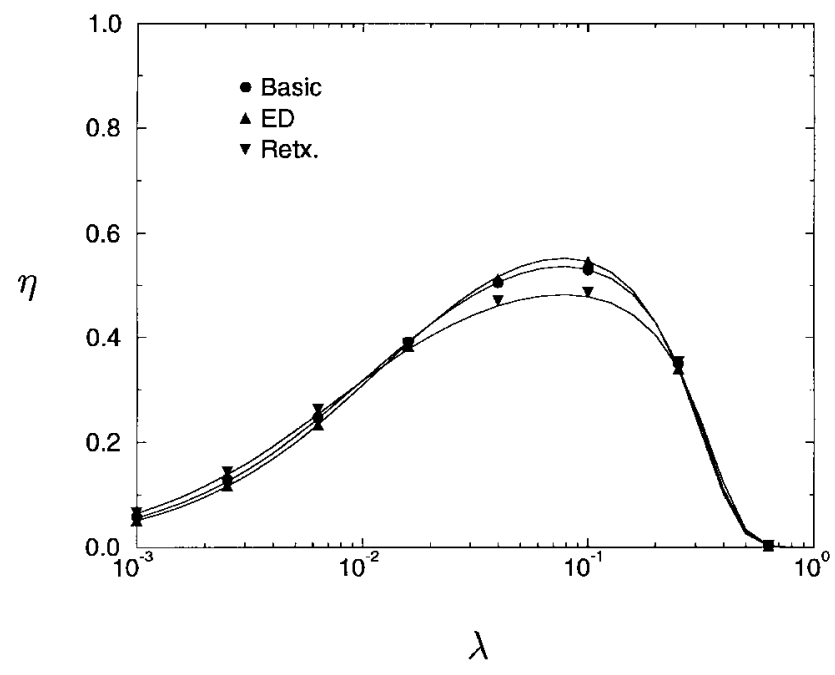

Fig. 7. Throughput $\eta$ versus message arrival rate $\lambda . N=10, g_{d}=0.1$, $f_{D} T=0.02, F=5 \mathrm{~dB}$, no capture, error-free feedback, one-slot propagation and processing delay.

because of the termination of data transmission following a data packet failure, the data packet success state and the data packet failure state in the basic protocol are to be expanded into four different states, namely, data packet success state following a data packet failure (state 6), data packet success state not following a data packet failure (state 7), data packet failure state following a data packet failure (state 8), and data packet failure state not following a data packet failure (state 9). Thus, a total of nine different states are needed to adequately describe the $E D$ protocol state space. The state transition probability matrix for the $E D$ protocol with one-slot propagation and processing delay $P_{\mathrm{ED}}^{(d)}$ can be written as

$$
\mathbf{P}_{\mathrm{ED}}^{(d)}=\left[\begin{array}{ccccccccc}
X_{0} & X_{1} & X_{2} & 0 & 0 & 0 & 0 & 0 & 0 \\
0 & 0 & 0 & 1 & 0 & 0 & 0 & 0 & 0 \\
0 & 0 & 0 & 0 & 1 & 0 & 0 & 0 & 0 \\
0 & 0 & 0 & 0 & 0 & 0 & X_{7} & 0 & X_{8} \\
X_{0} & X_{1} & X_{2} & 0 & 0 & 0 & 0 & 0 & 0 \\
X_{0} & X_{1} & X_{2} & 0 & 0 & 0 & 0 & 0 & 0 \\
g_{d} X_{0} & g_{d} X_{1} & g_{d} X_{2} & 0 & 0 & 0 & X_{3} & 0 & X_{4} \\
X_{0} & X_{1} & X_{2} & 0 & 0 & 0 & 0 & 0 & 0 \\
g_{d} X_{0} & g_{d} X_{1} & g_{d} X_{2} & 0 & 0 & X_{5} & 0 & X_{6} & 0
\end{array}\right]
$$

and the throughput of the $E D$ protocol is obtained as

$$
\eta_{\mathrm{ED}}^{(d)}=\pi_{6}+\pi_{7}
$$

Finally, 12 different states are needed to describe the retransmission protocol state space. Because the slot next to the successful last data packet slot is forcefully wasted in the retransmission protocol, the idle state is expanded into two separate states, namely, idle state following a data packet failure (state 1), and idle state not following a data packet failure (state 2). States 3-6 in the retransmission protocol state space are the same as states 2-5 of the basic and ED protocols' state space. Also, the data success and data failure states in the basic protocol need to be expanded into six 


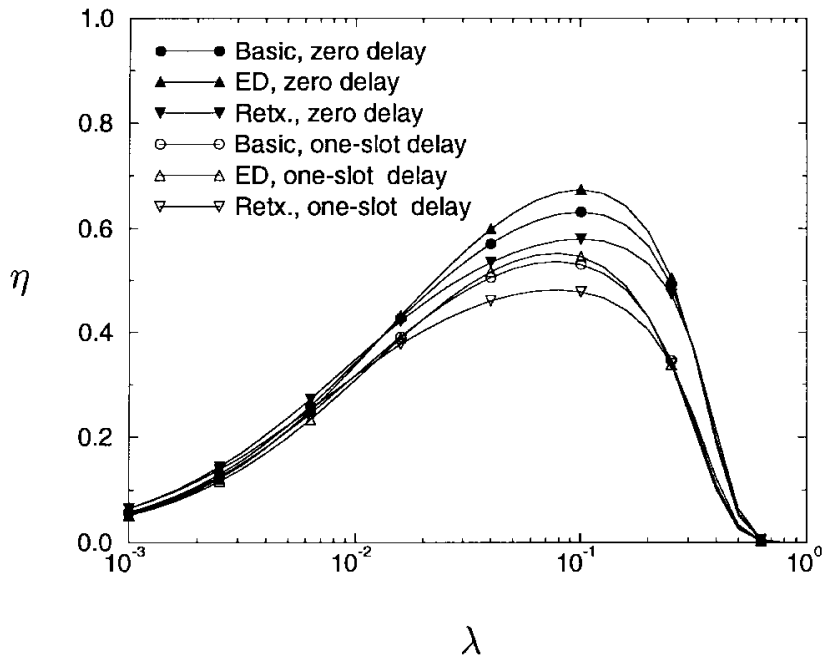

Fig. 8. Throughput $\eta$ versus message arrival rate $\lambda . N=10, g_{d}=0.1$, $f_{D} T=0.02, F=5 \mathrm{~dB}$, no capture, error-free feedback, zero and one-slot propagation and processing delay.

different states, namely, data packet success state: a) following a data packet failure (state 7); b) following an idle (state 8); and c) following neither data packet failure nor idle (state 9); and data packet failure state: a) following a data packet failure (state 10); b) following an idle (state 11); and c) following neither data packet failure nor idle (state 12). With these 12 different states, the state transition probability matrix for the retransmission protocol with one-slot propagation and processing delay $P_{\text {retx }}^{(d)}$ can be written as in (32), shown at the bottom of the page, where $X_{9}=q(1-q)+(1-q) p$ and $X_{10}=q^{2}+(1-q)(1-p)$. The throughput of the retransmission protocol is obtained as

$$
\eta_{\mathrm{retx}}^{(d)}=\pi_{7}+\pi_{8}+\pi_{9}
$$

The throughput performance of the basic, ED, and retransmission protocols with one-slot propagation and processing delay as computed by solving (28), (30), and (32) are plotted in Fig. 7. The parameters used for these plots are $N=10$, $g_{d}=0.1, F=5 \mathrm{~dB}, B \rightarrow \infty$, and $f_{D} T=0.02$. The results generated by explicit simulations without any

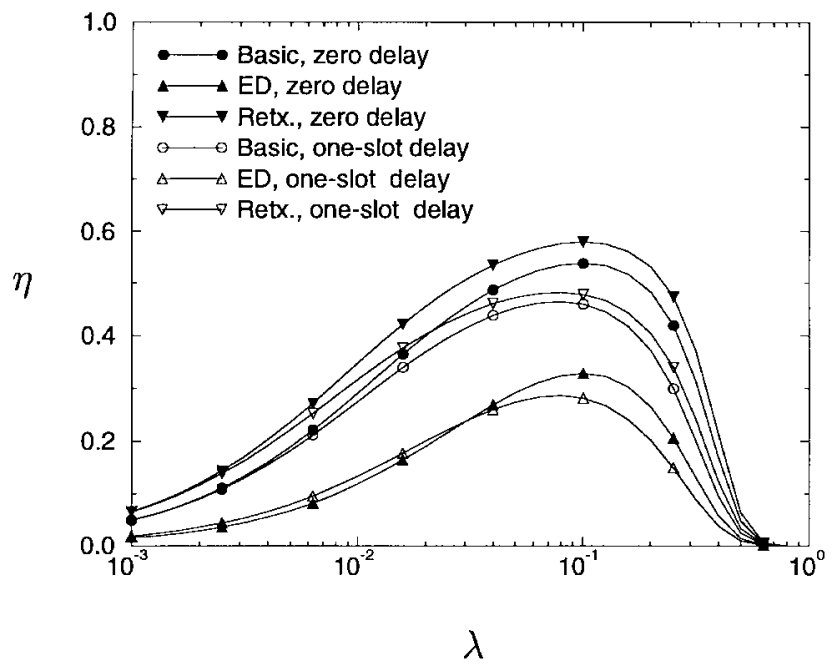

Fig. 9. Throughput $\eta$ versus message arrival rate $\lambda . N=10, g_{d}=0.1$, $f_{D} T=0.64, F=5 \mathrm{~dB}$, no capture, error-free feedback, zero and one-slot propagation and processing delay.

simplifying assumption are also plotted (in markers). In the analysis, header success events in vulnerable slots are ignored, whereas in the simulations, the event of a header success in the vulnerable slot and subsequent data packet transmission is allowed. It is seen from Fig. 7 that both analysis and simulation results agree very well for all three protocols. A comparison of the throughput performance of the three protocols for zero- and one-slot propagation and processing delay is provided in Figs. 8 (for slow fading, $f_{D} T=0.02$ ) and Fig. 9 (for fast fading, $\left.f_{D} T=0.64\right)$. The one-slot propagation and processing delay case is typically found to result in about $13 \%-20 \%$ degradation in the maximum throughput compared to the zero propagation and processing delay case.

\section{CONCLUSION}

We analyzed the throughput performance of a wireless media access protocol, taking into account the effect of correlated multipath channel fading, capture, and propagation and processing delays. The paper mainly focused on analyzing the effect and exploitation of the channel correlation. For efficient access on the uplink, the protocol made use of

$$
\mathbf{P}_{\text {retx }}^{(d)}=\left[\begin{array}{cccccccccccc}
0 & 0 & 0 & 0 & 0 & 0 & 0 & X_{9} & 0 & 0 & X_{10} & 0 \\
0 & X_{0} & X_{1} & X_{2} & 0 & 0 & 0 & 0 & 0 & 0 & 0 & 0 \\
0 & 0 & 0 & 0 & 1 & 0 & 0 & 0 & 0 & 0 & 0 & 0 \\
0 & 0 & 0 & 0 & 0 & 1 & 0 & 0 & 0 & 0 & 0 & 0 \\
0 & 0 & 0 & 0 & 0 & 0 & 0 & 0 & X_{7} & 0 & 0 & X_{8} \\
0 & X_{0} & X_{1} & X_{2} & 0 & 0 & 0 & 0 & 0 & 0 & 0 & 0 \\
0 & 0 & 0 & 0 & 0 & 0 & 0 & 0 & p & 0 & 0 & 1-p \\
0 & 1 & 0 & 0 & 0 & 0 & 0 & 0 & 0 & 0 & 0 & 0 \\
0 & g_{d} & 0 & 0 & 0 & 0 & 0 & 0 & X_{3} & 0 & 0 & X_{4} \\
0 & 0 & 0 & 0 & 0 & 0 & 1-q & 0 & 0 & q & 0 & 0 \\
1 & 0 & 0 & 0 & 0 & 0 & 0 & 0 & 0 & 0 & 0 & 0 \\
g_{d} & 0 & 0 & 0 & 0 & 0 & X_{5} & 0 & 0 & X_{6} & 0 & 0
\end{array}\right]
$$


the uplink channel status information, which was conveyed to the mobiles through a busy/idle flag broadcast on the downlink. A first-order Markov model was used to describe the packet success/failure process on the correlated Rayleighfading channel. A closed-form expression for the throughput was derived by modeling the system as a Markov chain. The analytical results obtained through the first-order Markov approximation of the channel were compared to those obtained from an i.i.d. channel model. The Markovian-fading channel model provided better performance results than the i.i.d. channel model. Simulations showed that a first-order Markov approximation of the fading process is quite accurate. An enhanced protocol with an error detect feature, taking advantage of the channel memory, was proposed and shown to improve performance under slow-fading conditions. The protocol with a retransmission feature to recover erroneous data packets was found to perform best under fast-fading conditions. Further, a parameterized retransmission strategy which allows a maximum of two or three retransmissions was shown to result in good performance over the range of mobile speeds of interest. The header packet capture phenomenon was shown to result in about $11 \%-18 \%$ improvement in system throughput under slowfading conditions. A simplified analysis was carried out to show the effect of nonzero propagation and processing delay on the throughput performance of the access protocols. A propagation and processing delay of one slot duration was shown to result in about $13 \%-20 \%$ degradation in maximum throughput compared to zero propagation and processing delay. Finally, it is noted that priority and fairness are important issues in access protocols. Extensions of the current analysis to address the priority and fairness issues are left for future investigation.

\section{REFERENCES}

[1] N. D. Wilson, R. Ganesh, K. Joseph, and D. Raychaudhuri, "Packet CDMA versus dynamic TDMA for multiple access in an integrated voice/data PCN," IEEE J. Select. Areas Commun., vol. 11, pp. 870-884, 1993.

[2] F. Borgonovo, M. Zorzi, L. Fratta, V. Trecordi, and G. Bianchi, "Capture-division packet access for wireless personal communications," IEEE J. Select. Areas Commun., vol. 14, pp. 609-623, May 1996.

[3] X. Qiu and V. O. K. Li, "Dynamic reservation multiple access (DRMA): A new multiple access scheme for personal communication system," Wireless Networks, vol. 2, pp. 117-128, June 1996.

[4] R. H. Clarke, "A statistical theory of mobile radio reception," Bell Syst. Tech. J., vol. 47, pp. 957-1000, July 1968.

[5] W. C. Jakes, Jr., Microwave Mobile Communications. New York: Wiley, 1974.

[6] H. S. Wang and P. C. Chang, "On verifying the first-order Markovian assumption for a Rayleigh fading channel model," IEEE Trans. Veh. Technol., vol. 45, pp. 353-357, May 1996.

[7] M. Sajadieh, F. R. Kschischang, and A. Leon-Garcia, "A block memory model for correlated Rayleigh fading channels," in Proc. IEEE ICC'96, Dallas, TX, June 1996, pp. 282-286.

[8] M. Zorzi, R. R. Rao, and L. B. Milstein, "On the accuracy of a firstorder Markov model for data transmission on fading channels," in Proc. IEEE ICUPC'95, Tokyo, Japan, Nov. 1995, pp. 211-215.

[9] A. Chockalingam, L. B. Milstein, and M. Zorzi, "Performance of a wireless media access protocol on a Markovian fading channel," in Proc. IEEE GLOBECOM'96, London, U.K., Nov. 1996, pp. 1769 1773.

[10] D. Bertsekas and R. Gallager, Data Networks. Englewood Cliffs, NJ: Prentice-Hall, 1987.
[11] B. Ramamurthy, A. A. M. Saleh, and D. J. Goodman, "Perfectcapture ALOHA for local radio communications," IEEE J. Select. Areas Commun., vol. SAC-5, pp. 806-814, June 1987.

[12] M. Zorzi and R. R. Rao, "Capture and retransmission control in mobile radio," IEEE J. Select. Areas Commun., vol. 12, pp. 1289-1298, Oct. 1994.

[13] A. Chockalingam, W. Xu, M. Zorzi, and L. B. Milstein, "Throughputdelay analysis of a multichannel wireless access protocol in the presence of fading and capture," submitted for publication.

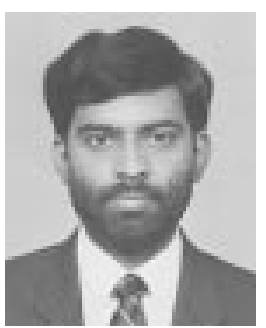

A. Chockalingam (S'92-M'93) was born in Rajapalayam, India. He received the B.E. (hons.) degree in electronics and communication engineering from the P.S.G. College of Technology, Coimbatore India, in 1984, the M.Tech. degree with specialization in satellite communications from the Indian Institute of Technology, Kharagpur, India, in 1985 and the Ph.D. degree in electrical communication engineering from the Indian Institute of Science, Bangalore, India, in 1993. His Ph.D. thesis work involved design and performance evaluation of media access protocols for wireless networks.

During 1986-1993 he was with the Transmission Research and Development Division, Indian Telephone Industries, Ltd., Bangalore, India, where he was involved in a range of $\mathrm{R} \& \mathrm{D}$ projects including VSAT networks using TDMA and CDMA, forward-error correction, and variable-rate PSK modem implementations using ASIC and DSP technologies. From December 1993 to May 1996 he was a Postdoctoral Fellow/Assistant Project Scientist with the Department of Electrical Communication Engineering, University of California-San Diego, La Jolla, CA, where he conducted research in CDMA wireless communications. He was a Communication Systems Consultant to CommQuest Technologies, Inc., Encinitas, CA, during 1994-1995. Since May 1996 he has been with Qualcomm, Inc., San Diego, CA, where he is currently a Manager and a Senior Systems Engineer, working in the area of modeling and performance evaluation of DS-CDMA wireless communication systems. His research interests lie in the area of wireless networks and digital communication systems.

Dr. Chockalingam is a recipient of the Communication Devices India, Ltd. (CDIL) Award for a paper published in the IETE Journal.

Michele Zorzi (S'89-M'95-SM'98), for photograph and biography, see p. 356 of the March 1998 issue of this Transactions.

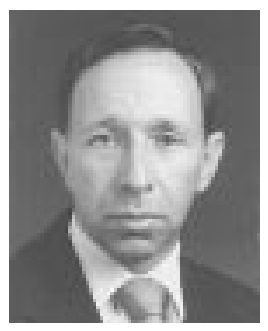

Laurence B. Milstein (S'66-M'68-SM'75-F'85) received the B.E.E. degree from the City College of New York, NY, in 1964, and the M.S. and $\mathrm{Ph} . \mathrm{D}$. degrees in electrical engineering from the Polytechnic Institute of Brooklyn, Brooklyn, NY, in 1966 and 1968, respectively.

From 1968 to 1974 he was with the Space and Communications Group, Hughes Aircraft Company, and from 1974 to 1976 he was with the Department of Electrical and Systems Engineering, Rensselaer Polytechnic Institute, Troy, NY. Since 1976 he has been with the Department of Electrical and Computer Engineering, University of California-San Diego, La Jolla, CA, where he is a Professor and former Department Chairman, working in the area of digital communication theory with special emphasis on spread-spectrum communication systems. He has also been a Consultant to both government and industry in the areas of radar and communications.

Dr. Milstein was an Associate Editor for Communication Theory for the IEEE Transactions on Communications, an Associate Editor for Book Reviews for the IEEE TRANSACTIONS ON INFORMATION THEORY, and an Associate Technical Editor for the IEEE Communications Magazine. He is currently the Editor-in-Chief of the IEEE Journal on SELECTED AREAS IN Communications. He was the Vice President for Technical Affairs in 1990 and 1991 of the IEEE Communications Society, and has been a member of the Board of Governors of both the IEEE Communications Society and the IEEE Information Theory Society. $\mathrm{He}$ is also a member of Eta Kappa $\mathrm{Nu}$ and Tau Beta Pi. 


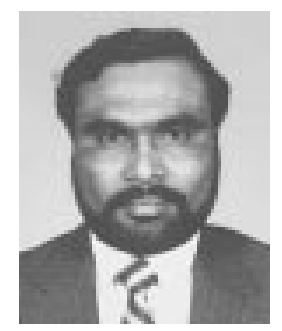

Pallapa Venkataram received the M.Sc. degree in mathematics from Sri Venkateswara University, Tirupathi, India, in 1973, and the Ph.D. degree in information sciences from the University of Sheffield, U.K., in 1986.

$\mathrm{He}$ is currently an Associate Professor of electrical communication engineering with the Indian Institute of Science, Bangalore, India, where he is involved in wireless access research and protocol engineering. He was with Hindustan Aeronautical Limited, Bangalore, India, as a Deputy Manager (computers) from 1980 to 1982, where he worked mainly on systems design for financial and material management systems. He was a Visiting Scientist at the University of Maryland, Baltimore, and at the University of Montreal, Montreal, P.Q., Canada, in 1992. In 1995 he was a Visiting Scientist at the University of Enschede, The Netherlands, and a Visiting Professor at the SK University, Anantapur, India. In 1998 he spent six months with NORTEL, Canada, on sabbatical. He serves on several advisory committees and frequently consults to government and industry. His current research interests are in the areas of communication protocols, wireless internet, and AI applications in communication networks and multimedia systems. $\mathrm{He}$ has published over 70 papers and he coauthored a chapter in the recently published book Mobile Multimedia Communications. He holds a Distinguished Visitor Diploma from the Orrego University, Trujillo, Peru. He received a Communication Devices India Ltd. (CDIL) Award for a paper published in the IETE Journal.

Dr. Venkataram has served in various capacities in many IEEE and ICCC conferences and workshops. He is a Fellow of the Institution of Electronics and Telecommunication Engineers (IETE) and a senior member of IEEE Computer Society. 